\title{
Model-based definition capabilities and its impact on industrial productivity
}

\author{
Emmanouil Varitis ${ }^{1, *}$, Konstantinos Rinos ${ }^{1}$, and Nikolaos Kostis $^{2}$ \\ ${ }^{1}$ University of Western Macedonia, Department of Mechanical Engineering, 50100 Kozani, Greece \\ ${ }^{2}$ University of Patras, Department of Mechanical Engineering and Aeronautics, 26500 Patras, Greece
}

\begin{abstract}
The need for optimization of the product manufacturing cycle and data management resulted in the creation of the model-based definition technology. The beneficial impact in most processes (CAD, CAE, CAPP, CAM, etc.) of a product manufacturing cycle, made the MBD technology implemented in various industries. The following paper has the purpose of highlighting the benefits and limitations of the current MBD capabilities and makes its impact on the industrial collaboration known by summarizing the communication file formats, which support this technology. Special interest is attributed to lightweight collaboration files, especially 3D PDFs, which are capable to deliver information and data to the domains of a company inside or outside of the manufacturing cycle and support all the necessary downstream activities of the product.
\end{abstract}

\section{Introduction}

It has always been industry's intention to create economical, accurate, reliable, and timesaving methods of distributing product data across its sub-branches such as design, manufacturing, managing departments, etc. The first big step was the digitalization of information like 2D schematics, which enabled companies to fasten-up their designing processes by eliminating the need for hand sketching and therefore decreasing errors originating from human personnel [1]. However, its impact on data distribution and minimization of the necessity for supplementary documents is not exactly spot on. Thankfully a very promising candidate emerges in the name of model-based definition (MBD). The resourceful idea of implementing all the important information on a 3D model without the infringement of the principles of mechanical design has positive effects on the product's life-cycle management (PLM) and especially in the manufacturing processes [2]. The following research summarizes the evolution and the capabilities of MBD technology and points out the limitations of its current use. Furthermore, this paper divulges the impact of MBD technology in industrial collaboration and product data management (PDM), based on the interoperability of the existed exchange information formats.

\footnotetext{
*Corresponding author: evaritis@uowm.gr
} 


\section{MBD evolution and research overview}

The Model-Based Definition (MBD) technology is considered as an innovative tool for the digital definition of a product, as well as a helpful guide for its subsequent manufacturing processes. Besides, this technology is not a current discovery but, it is originated in the 1990s when the aerospace industry and specifically the Boeing used it as a tool for the reduction of the aircraft production cycle [3]. As soon as possible (1997), the American Association of Mechanical Engineers (ASME), in cooperation with the Boeing Company, started its research for this new methodology and by July 2003 developed the standard "ASME Y14.41-2003, Digital Product Definition Data Practices" [2]. This standard destined for the use of the engineering team of the American Department of Defense (DoD) to fully define the requirements and the best practices for the manufacturing of its products (the latest version ASME Y14.41-2019). For the same reason, ASME launched in February 2009, the standard "ASME Y14.5-2009, Dimensioning and Tolerancing", which has been revised to its current version in 2018. This standard operates as guidance for the establishment of rules and requirements of the implementation of dimensions and tolerances. MBD technology uses both of these standards for the integrated definition of the manufacturing intent of a part or assembly. At the same time, European countries wanted to include this technology to their international standards, developed in 2006 the standard "ISO 16792-2006, Technical Product Documentation - Digital Product Definition Data Practices", to specify the operational requirements of the 3D model definition data, including the engineering data of 2D drawing (the latest version ISO 16792-2015). These standards assist in straddling the chasm between the distinguished use of MBD technology and its general use from a variety of industrial domains. Specifically, MBD is now fully adopted by the engineering software industry as the main idea for the development of a variety of software programs SOLIDWORKS MBD, CATIA MBD, PTC CRO MBD, etc.

Despite the current capabilities of MBD, there is a necessity for optimization and implementation in more industrial fields. Therefore, this technology has been the point of interest in many kinds of research over the last years. Quintana et al. achieved to define the limitations [4], which restrict the full adoption of MBD as the resource of information for the product life cycle. Based on their research, there are still many industrial requirements that should be overcome, but according to their benefits, MBD has the potentials to replace the 2D drawings in the future. Furthermore, this technology is considered a useful tool for inspection and manufacturing processes. As a consequence, Neng Wan et al. introduced two different methods (forward and reversed) for the creation of a 3D machining process based on the MBD technology [1]. These methods enhance the efficiency of the process planning with the combination of machining knowledge and model information in the same document. Nevertheless, MBD documents are great resources for commerce information (resources, marketing, management, finance, etc.) of a product and they can improve the collaboration between interior (branches inside the company) and exterior (partners, customers, etc.) teams. For that reason, Hartman et al. proposed a framework for the evaluation of different collaborative MBD product representations based on four different data formats [5]. This framework includes the necessary criteria of the workflows, which included in a product life cycle insight a Model-Based Enterprise (MBE) [7]. 


\section{MBD technology and capabilities}

\subsection{Concept and applications}

The Model-Based Definition (MBD) is a revitalizing technology that utilizes 3D models as the principal carrier of all vital information of the overall product life cycle. The main idea is to establish 3D models as the main source of information for processes such as design, production, technical documentation, management, etc. Specifically, MBD data sets are composed of 3D geometry, dimensions, tolerances, and manufacturing annotations [6]. A demonstration of dimensions viewed in a $3 \mathrm{D}$ model is shown in figure 1. Additional information regarding the bill of materials (BOM), technical information, and production properties can be implemented. Moreover, it provides the fluency of automation regarding repetitive tasks by standardizing data [8]. The enrichment of 3D models with drawing annotations and geometrical-topological information optimize their implementation in CAM machining processes, mitigating this time-consuming procedure [2]. Besides that, the avoidance of the error-prone process of converting 3D data to $2 \mathrm{D}$ drawings is of great importance. From a marketing perspective, the $3 \mathrm{D}$ visualization of the final product is useful for promoting and communicating with customers [4]. Consequently, MBD can have a central and pivotal role in managing and distributing data across many brunches avoiding excess, supplementary, and duplicated documentation. As a sequence time and cost of engineering procedures are decreased.

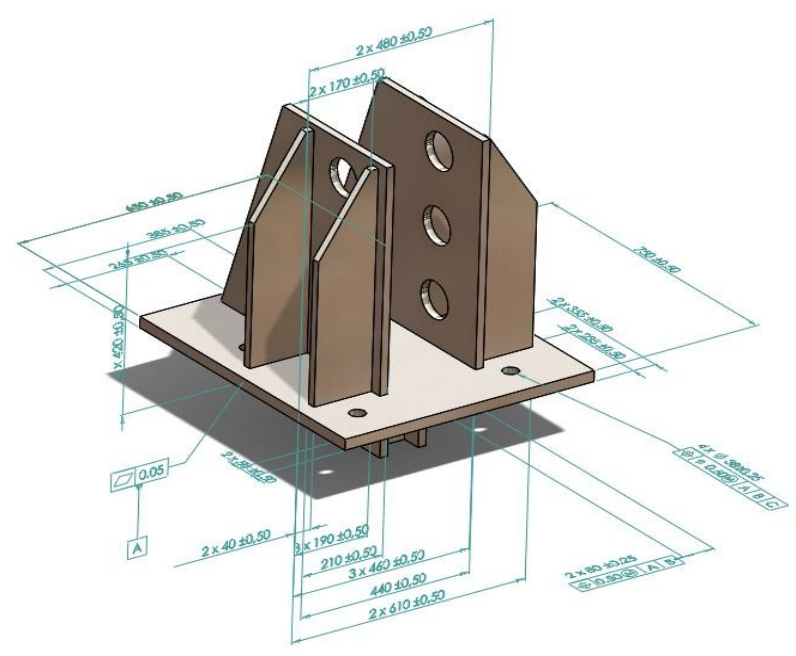

Fig. 1. MBD dimensions and tolerances implementation in 3D model.

\subsection{Benefits}

The beneficial impact of MBD technology does not pertain only to its capabilities per se. Firstly, the ease of manipulation of the model in cooperation with the well-structured CAD software environment, lead in the deep understanding of even the most complex geometries and optimize the interaction between the user and the model. Moreover, modern MBDCAD software provides automation in dimensioning, positioning, tolerancing, etc. enabling the user to use commands like auto-dimensioning or classify groups of features, which have the same properties. Besides that, the use of some automation in complex geometries or 
assemblies may cause many problems, but its implementation in simple parts can improve the productivity of the designer as well as the efficiency of the model. Furthermore, MBD technology aims the understating of the design intention of the model and improves the interpretation of non-designer users [4]. Considering this, the attached information on the model operates as a supplementary factor for the subsequence processes of the $3 \mathrm{D}$ model. For instance, the feature-recognition process is a fundamental step for the manipulation of the 3D models in neutral files form and exists in every process (CFD, CAPP, CAM, CMM, etc.) of the product manufacturing cycle after the design [1,9]. Therefore, MBD technology aims this process by providing important information, like the existence of a number of holes or chamfers with the same dimensions, and prevents the user from doing it manually. Another advantage of the cooperation between MBD technology and modern CAD software is the automatic recognition of standardized features [10]. Standards like ASME Y14.41, ASME Y14.5, and ISO 16792 are already installed in the CAD software, and therefore, the MBD users do not need to spend valuable time for dimensioning standardized features (holes, metric threads, etc.) [2]. Last but not least, the combination of 2D drawings and $3 \mathrm{D}$ models in a single document, as well as the continuous updating of this document for changes, resulting in the improvement of the PDM process. Consequently, the easy in managing and storing has a great impact on its later use from departments inside or outside of the product manufacturing cycle (sales, technical support, marketing, etc.).

\subsection{Limitations and fields of improvement}

Despite all the privileges, MBD has to offer there are still handicaps to overcome in order to take a form that will make feasible its smooth immersion in industry. Considering the benefits that occur from the model-based definition method, its adaption from the industry seems to be highly possible. Namely, access to CAD software must be secured for diverse users across the conceptualization, design, manufacturing, quality control, and promotion phase of a product. Printing MBD files is not suggested, due to its nature, it is only readable in 3D environments. Consequently, not only a need for new hardware and software acquirement might arise but also a portion of personnel will have to get acquainted with 3D modeling manipulation. Moreover, highly important is the fact that different portions of the design process have personalized data requirements. To this point, this is arranged by the creation of different types of 2D drawings (assembly, detail, layouts, manufacturing, etc.). Therefore, MBD has to be able to deliver the necessary data to each subgroup while allowing them to use it for their specific tasks without actually modifying it [4]. Taking into account the design of highly complex solid parts, the establishment of international standards will guarantee compliance under strict rules for implementing information to the 3D document properly and universally reducing the risk of misconceptions. For instance, in cases of complex assemblies, and surfaces even experienced users might have to deal with the highly demanding process of defining dimensions as well as extensive and dense engineering data correctly in order to accomplish a successful and efficient inspection. Nevertheless, the existing automation for dimensioning can facilitate this process, but there is still a necessity for additional processing from the user, such as the positioning of the dimensions in proper views for the better interpretation of the model. However, MBD is 
still a more efficient and simple way for dimensioning, than the traditional 2D drawings, which require technics like model decomposition in its sub-bodies.

\section{Efficiency in collaboration}

The process of product development depends on various factors such as the complexity of manufacturing, the material, the size, the existence of electronic parts, etc. As a result, the final form of the product originates from the cooperation of different branches from the same or separate company, which uses a variety of software and types of data. Speaking about a model-based enterprise (MBE), the MBD technology accompanies the product form the design process until its deposition for future rehashing. Considering that, this technology is supported by various file formats, which include different information or representations of the product for a variety of uses. Native MBD files can only be shared between software (CAD, CAM, PDM, etc.), which use the same methods for data management and storage, but they allow the user to make changes to the model directly from its design history [5]. Furthermore, neutral MBD files like STEP AP 242 "Managed Model-Based 3D Engineering" include data for inspection, manufacturing, engineering visualization, supplier information, etc., which enables the collaboration between different software (SOLIDWORKS, CATIA, AUTOCAD, etc.), with the cost of possible data loses (design tree, mates, etc.) [11].

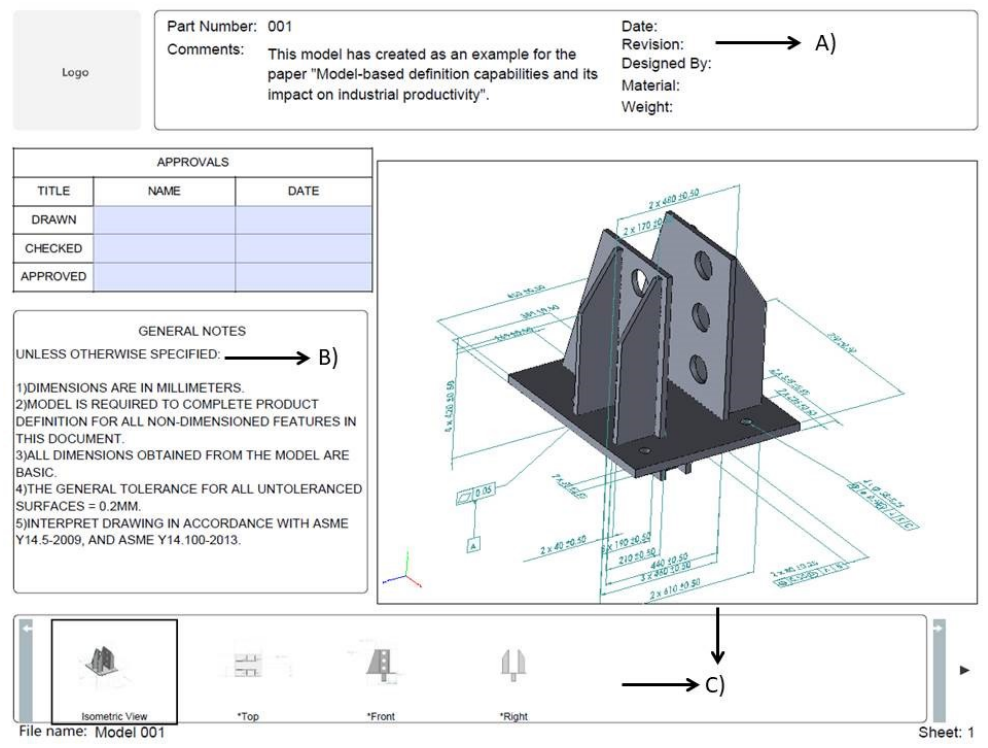

Fig. 2. Detailed MBD data exchange 3D PDF format.

Lightweight formats (3D PDF, 3D XML, JT, etc.) are another category of files, which have as purpose to share information and data of a 3D model, without the typical cost of advanced hardware and software [5]. The main difference between these files and the other two categories is that the Lightweight files contain information for the visualization of the model and are destined to enhance the collaboration between the labor force and not as a linking tool between CAD software. One of the most useful files of this category is the 3D 
PDF format. These files enable the user to create 3D views of the MBD model or assembly and export them, including important information for the downstream activities of the model. As an example, figure 2 demonstrates a 3D PDF, which operates as an animated environment for the visualization of a 3D MBD model and its properties. This file includes three different categories of information. The first category (A) includes general information about the creation and revision of the part, as well as some physical properties. Category (B) is a section where the user can add important complementary information regarding various aspects of design. For example, in the case of assembly, notes which address mechanical joints and the way different parts are connected, can ease and guide the process. Moreover, category (C) enables users to have an integer visualization and 3D manipulation of the model. Also, 3D PDF provides useful tools such as section view, measurement, changing transparency etc. that assist the user to achieve better model inspection. Last but not least, these types of formats have the option of including different pages with various information like the bill of material for the supplier chain, manufacturing notes, etc.

\section{Conclusions}

MBD technology's capabilities have been increased drastically in recent years. They have evolved in a form capable of optimizing and supporting product life cycle management (PLM). With the current interoperability of data formats that support this technology, it is expected that MBD will become the main source of product information not only in the manufacturing process but also in other divisions of the industry. Moreover, this research enlightens the advances in collaboration through formats like 3D PDF files based on information (dimensioning, bill of materials) that can be distributed by them. Even though there are still aspects to improve, this technology has the credentials for further research due to its potential for improving manufacturing and industrial collaboration.

\section{References}

1. N. Wan, R. Mo, L. Liu, J. Li, Comp.In.Indu,65,537-549,(2014)

2. W. ZHU, M. BRICOGNE, A. DURUPT, S. REMY, B.LI, B.EYNARD, IFAC. Pap.On.Li, 49,485-490,(2016)

3. B. Friendland, R. Malone, J. Herrold, INCOSE. Int. Sym, 26, 386-398,(2016)

4. V. Quintana, L.Rivest, R. Pellerin, F.Venne , F. Kheddouci, Comp.In.Indu, 61, 497$508,(2010)$

5. N. Hartman, P. Rosche, K. Fischer, Int.Con.Prod.Lif.Rep.In.Prod.Lif.Wor, 388,424434,(2012)

6. Y. W. Huang, IOP Conf. Ser.: Mater. Sci. Eng. 616, (2019)

7. S. Frechette, CIRP.Int.Con.on.Man.Sys, (2011)

8. M. Alemanni, F. Destefanis, E. Vezzetti, Int.Jour.Adv.Manuf.Tec, 52,1-14,(2011)

9. L. Li, Y.Zhengd, M. Yange, J.Lengf, Z.Chengd, Y. Xieg, P. Jiange, Y. Mad, Rob.Com.Int.Man, 61, (2020)

10. W.D Li, S.K. Ong, A.Y.C. Nee, Com.Aid.Des, 34,849-868, (2002)

11. A.Sarkara, D. Šormaza, Int. Con. Flex. Aut. Int. Man, 38,513-523,(2019) 